Antropología Cuadernos de Investigación, núm. 14, julio-diciembre 2014, pp. 111-116

\title{
Entrevista a Remigio Cáceres con Edgar Báez, Peguche
}

Carolina Páez ${ }^{1}$

$\mathrm{M}$ isión Andina del Ecuador fue una institución que surgió dentro el Programa Indigenista Andino de la Organización Internacional del Trabajo a finales de 1950. $\mathrm{Su}$ principal objetivo era transformar las precarias condiciones de vida y trabajo de las poblaciones indígenas de las llamadas "comunidades libres". Este gran proyecto, que se implementó inicialmente en Bolivia y Perú, se ubicó a su vez dentro del proyecto nacional de integración de la población indígena a la sociedad nacional; razón por la cual estuvo adscrita al Ministerio de Previsión Social y Trabajo.

Los proyectos de la Misión se organizaron de acuerdo a ámbitos de acción, entre ellos estuvieron salud, producción, infraestructura y educación. Misión Andina realizó actividades hasta inicios de la década de 1970 cuando fue absorbida por varios ministerios en el marco de los gobiernos militares de ese momento.

Esta entrevista fue realizada en marzo de 2011, a Remigio Cáceres de la provincia de Imbabura. Él fue educador en diversos espacios educativos. En este relato, él cuenta su paso por la Misión Andina, así como su relevante trayectoria de educador.

Remigio: yo tengo una especialidad de idioma quichua, entonces yo he dado cursos en la universidad de Nariño en Pasto, Colombia, he prestado mis servicios durante 20 años en la Universidad Católica, aparte de lo que he estado de profesor en lo fiscal, mi nombre es Remigio Cáceres, profesor en servicio pasivo desde 1994, pero sin embargo cuando se requiere de alguna información yo estoy listo, pues por colaborar, siempre y cuando sea en beneficio social.

Carolina: por supuesto, es muy importante y creo que tenemos un problema ahorita, le voy a contar es difícil encontrar documentos, los archivos de los que hizo la Misión porque todo se ha quemado, se ha perdido, no hay y eso nos hace olvidar, y nuestro principal objetivo ahorita es luchar contra ese olvido, entonces parte de esta investigación es no solamente ver cómo funcionaba la Misión Andina, en su parte administrativa, organizativa sino como era la relación con la comunidad; quizá usted recuerda cómo llegaron, cuál fue la impresión que tuvieron, que les dijeron los personeros de la Misión Andina.

1 Esta entrevista se realizó en el marco de la investigación "Mujeres indígenas y Estado: la Misión Andina en Ecuador 1950 - 1975", coordinada por Mercedes Prieto y financiada por el Fondo de Desarrollo Académico de la sede Ecuador de FLACSO. Carolina Páez, profesora de la PUCE. Correo electrónico: lacaropaez@yahoo.com 
Remigio: bien, creo que más o menos por el año de 1961, cuando trabajaba en la comunidad de Paniquimra que se encuentra tras de Imbabura, que pertenece a la parroquia La Esperanza del cantón Ibarra, presté mis servicios desde 1958 y desde ahí en el 61 Misión Andina entró a trabajar en las comunidades. Realmente Misión Andina ha cumplido una función social de apoyo hacia las comunidades, tal es el caso que cuando yo estaba en Paniquimra, ya teníamos que defender a la raza indígena en vista que había en esa época de parte de señor Carlos Puga, propietario de la hacienda de Cuchicaranqui, de este sector de la Florida; entonces ahí los indigenas fueron maltratados, esclavizados.

En esa época Misión Andina [y] con quien habla tuvimos que seguir un juicio para sacar un camino que permita entrar a la comunidad porque en ese entonces o antes de eso había solamente un sendero que la gente tenía que ocupar por ahí, pero sin embargo el dueño de hacienda tenía que quitar alguna prenda para tener trabajando lunes, martes, miércoles y jueves cuatro dias, entonces Misión Andina en ese entonces, pues ya conjuntamente con los profesores había ese apoyo para seguir en la apertura del camino, con el Ministerio de obras Públicas, y se logró un camino carrozable a Paniquimra, lo cual ahora está en servicio no solo a (Paniquimra) sino también a otra comunidad más arriba que se llama el (Abra); entonces nosotros sabíamos estar trabajando con Misión Andina y los recursos que obtenía Misión Andina siempre en función en ayuda hacia las comunidades, por eso es que nosotros también conscientemente ya que otra institución a las comunidades en esa época no entraba no, entonces Misión Andina si entraba hacia las comunidades y ha dejado en muchas comunidades construyendo escuelas.

De ahi de la comunidad de (Paniquimra) me tuve que bajar a la comunidad de Chiriguasi. En la comunidad de Chiriguasi construyó la escuela porque tanto en (Paniquimra) como en Chiriguasi era una casa arrendada en situaciones deplorables que no podía el profesor, realmente por más que quería enseñar no reunía en el aspecto pedagógico, entonces hemos hecho gran sacrificio el profesor, sin una tiza, sin nada pero ya cuando la Misión Andina ya estaba, hemos recurrido que nos de tiza, papel, papelitos que necesitamos para el material didáctico de la escuela, entonces en la comunidad de Chiriguasi construyó una escuela grande con dos viviendas para profesores, con una canchita para los niños, para la distracción, para el deporte, para realizar ejercicio físicos, entonces poco a poco así Misión Andina fue entrando en las comunidades, apoyando y ayudando realmente hacia las comunidades.

En Chiriguasi por ejemplo; la comunidad en esa época en 1963, los comuneros de la hacienda La Magdalena ganaban 7 sucres diarios, los huasipungueros. Los huasipungueros de la comunidad de Chiriguasi ganaban tres sucres, entonces conversábamos con los comuneros para lograr que se cumpla en todos los huasipungueros el pago de acuerdo a la ley que era ya el pago de los siete sucres, entonces tuvimos que reunirnos y luego, en la Inspectoria Provincial de Trabajo fuimos y demandamos, entonces el arrendatario, que era de Cayambe, había ciertas amenazas pero no nos había hecho nada, trabajamos con las comunidades, respaldaba la comunidad, entonces igual ahi ya se le hizo pagar 7 sucres diarios a los huasipungueros de la comunidad de Chiriguasi.

Asi ha habido algunos problemas que realmente tuvieron en las comunidades y nosotros hemos logrado conjuntamente con la Misión Andina que coordinábamos con las escuelas de Misión Andina para resolver muchos problemas que para ese entonces habia mucho abuso todavía de parte de los hacendados que obviamente nos dolía, nos dolía la esclavitud, la explotación de las comunidades indígenas...

Edgar: hay una anécdota muy interesante en esta comunidad que estabas diciendo, Remigio, los varones trabajan en la asistencia social en la hacienda en Cacho, y en la comunidad estaban solo las mujeres. Ellas preparaban la tierra para hacer los bloques, ellas trabajaban. Las mujeres y los hombres hacían la minga el domingo para hacer las máquinas para hacer los bloques el día sábado. Entonces fíjese cómo los interesó la educación que las mujeres salían a pisar el barro a prepararlo y los hombres hacían sábado y domingo a minga. Ahí fue director de escuela, de la primera escuela que se hizo completa en ese sector. 
Remigio: sí en verdad, para construir las escuelas en la misma comunidad se elaboraba los bloques para la construcción y siempre la comunidad participaba mediante mingas y realmente cuando el profesor cumplía a cabalidad, en lo que se refiere a la educación, los comuneros eran muy alegres, contentos y siempre colaboraban con cualquier cosa para mejorar, para adelantar a la escuela. Entonces, yo pasé en la comunidad de Chiriguasi ocho años, en la comunidad de Paniquimra pasé cinco años, total por ese sector estaba 13 años.

De ahi es que la Misión Andina y la Dirección Provincial de educación de Imbabura seleccionó a los profesores indígenas, a los más antiguos, para luego formar el colegio Fernandez Chávez de aquí de la comunidad de Quimchuqui; es decir, formados solo cuatro indígenas, fue la compañera Lola Vega, Luz María Chiza, Alberto Andrango que ahora es alcalde de Cotacachi, y quien habla: cuatro profesores. Fundamos ese colegio pero realmente ahi si Misión Andina nos ha aportado bastante con alimentos para el internado del colegio, fue internado de las diferentes comunidades visitantes, hasta vinieron del Inca, entonces ahi había un gran aporte en lo que se refiere a la alimentación, que esa alimentación sirve para el internado para apoyar a los estudiantes que vivían distantes.

Carolina: ¿Usted dónde se formó como maestro?

Remigio: mi formación es cuatro años, primero, segundo, tercer, cuarto grado aquí en la parroquia de Iluman. No había quinto y sexto, tuve que ir a terminar en Otavalo en la escuela Ulpiano Pérez Quiñonez; de ahí estaba en primero, segundo, tercer, cuarto, curso en el colegio de Otavalo y luego fui al colegio normal Juan Montalvo quinto y sexto curso. Ventajosamente mi papá tenía relaciones con muchos abogados, médicos en Quito, era el quinto velasquizmo en 1955 cuando entonces fue ministro de gobierno Manuel Araujo Hidalgo, entonces era muy amigo de mi papá, él habló con [este] para que a su vez él hable con el ministro de educación para que me consiguiera una beca, entonces se logró conseguir una beca de 120 sucres mensuales para poder concluir quinto y sexto curso en el colegio Juan Montalvo de Quito.

Luego me vi conjuntamente con Alberto, había la oportunidad en la universidad Católica cuando se abren los cursos de especialización quichua los viernes y los sábados, concurríamos a las tres de la tarde, teníamos que estar en Quito, viernes y sábado teníamos que estar hasta las tres de la tarde. Para ser profesor en cualquier idioma teníamos que saber la estructura y la gramática del idioma español, pues si no se sabe la gramática en la lengua española pues, no podíamos conocer saber, también la estructura de la lengua quichua. Asi hemos trabajado. Luego fui rector del colegio Ingeniero Federico Páez en la ciudad de Otavalo. También trabajé ahí con mucha dedicación y responsabilidad para dejar lo que actualmente, ese colegio tiene 500 alumnos; entonces se levantó ese colegio. En 1993- 94 la universidad Católica de Ibarra suscribió un convenio con la universidad de Nariño de Pasto, Colombia, entonces me designó a mi para que fuera a dar curso en la universidad de Pasto, por un espacio más o menos de unos ocho meses, por el Putumayo colombiano, Puerto Asís también; he pasado bien con la Universidad de Nariño, Pasto, buena atención había.

Carolina: hace un momento usted mencionó que en las comunidades habían algunos problemas, uno de esos problemas era el trato o las condiciones que tenían.

Remigio: había problemas de que las comunidades no teníamos agua, entonces la Misión Andina por ejemplo, de aqui en las faldas del Imbabura tuvo que realizar excavaciones para traer el agua que más o menos era en ese entonces 4 o 5 litros por segundo para la parroquia de Iluman y sus comunidades, porque actualmente la parroquia de Iluman cuenta más o menos con unos 13 mil habitantes, considero que para mi la parroquia de Iluman en el momento actual tiene más relación comercial. Yo fui por más o menos 30 años dirigente de esta comunidad, he trabajado, sacado calles. He trabajado con el Consejo Provincial el adoquinado, hemos levantado la escuela, en la gestión cuando yo era presidente de la Junta parroquial y así se ha hecho muchas cosas, cuando no ha funcionado aqui el teniente político hemos tenido que luchar con teniente político para cambio. El señor Edgar Báez es conocedor que yo en calidad de presidente del comité promejoras tuvimos que enfrentar hasta con los profesores en vista de 
falta de cumplimiento de planificación y del horario, entonces me dolía bastante porque la gente indígena podian reclamar; en cambio yo como presidente de la Junta del comité promejoras tuve que poner un poco en regla a los profesores, con el señor Báez que fue supervisor en aquel entonces con el señor director de educación de ese entonces fue el doctor Benalcazar.

Entonces vea, hemos hecho algunas cosas pero conscientemente de lo que uno se ha formado, desde abajo hacia arriba en situaciones tan miserables, que mi papá no contaba con los recursos económicos necesarios, había que hacer gran esfuerzo para acabar de estudiar y no había estudiantes en aquel entonces, era casi yo el único indígena; al menos cuando estaba en el Juan Montalvo era el único indígena entre los 900 estudiantes mestizos de ahí del normal, sin embargo ahi tuve un poco de dificultad a pesar de estar matriculado siempre a los indígenas casi no querían apoyar.

Pero sí, Misión Andina también apoyó a muchos indígenas para becarles; al Carlos Zambrano de Uyumbicho allá fueron a estudiar muchos indigenas, entonces si ha hecho bastantes cosas Misión Andina, entonces la formación mía ha sido tan difícil, dura creo que eso ha motivado en mí para respaldar, gestionar, ayudar hacia las comunidades, no solamente aqui porque en el año 84 tuve que en comisión de servicio, en ese entonces era León Febres Cordero el presidente, pero no porque era de León Febres Cordero si no porque le conocía al señor Plutarco Cisneros que era presidente del Municipio y logró conseguir como comisión de servicio para trabajar en las 25 comunidades por primera vez implementando los centros comunitarios infantiles, que viene a sustituir al jardín de infantes, en las comunidades no había entonces eso. He trabajado responsablemente, igualmente coordinando en el INNFA nacional en el Ministerio de Bienestar Social y el Municipio de Otavalo. Trabajé durante cuatro años en las comunidades implementando con los centros comunitarios infantiles, entonces también he tenido el gusto de servir a mi raza en las comunidades, para que poco a poco vayan orientándose, vayan conociendo que es importante la educación.

Carolina: había los problemas de agua, de caminos

Remigio: eso exactamente agua, caminos, educación, apoyar a las gentes que no tenían por ejemplo, que no tenían casita, haciendo los adobes o los bloques en las comunidades, el aparato tenía que ir de comunidad en comunidad, elaborando los bloques para que las personas pobres que no tenían casita con eso realicen casitas.

Carolina: ¿cómo transportaban la maquinaria?

Remigio: Misión Andina tenía transporte, para trasladar de una comunidad a otra, pero habia comunidades que estaban bastante, bastante conscientes para recibir ese apoyo y trabajar conjuntamente con la Misión Andina. Yo creo que ahora igual si es que nuevamente Misión Andina viniera a trabajar habría que seleccionar personal para que, en el momento actual siempre se está mirando el reloj para retirarse, en cambio Misión Andina trabajaba hasta altas horas de la noche en las comunidades, reuniones, para incentivar la importancia que tenía el agua, el camino, la educación, vivienda, especialmente la salud.

En salud también había la licenciada Hermosa que trabajaba, es decir, en Misión Andina estaba implementado para trabajar por salud, por educación, en general por desarrollo comunitario, entonces había el personal que tenía que trabajar en las comunidades. Siempre era necesario que la gente conozca, haga consciencia de las verdaderas necesidades que tiene la comunidad y que haya participación, y que no sea solo el que recibe, sino que también ellos participan para que sientan lo que ellos trabajaron también. En ese sentido, no ha habido tanto paternalismo, siempre en todas las comunidades habia mingas y mingas para cualquier trabajo que requiera la comunidad.

Carolina: ¿cómo organizaban las convocatorias, que les decían a las personas para que vayan?

Remigio: en la comunidad habia los cabildos, las comunas, ellas eran encargadas de hacer conocer cualquier problema que hay; el profesor y con la comuna se tenía que conversar para realizar reuniones, para realizar mingas. Entonces, habia una gran participación en mingas en esa época por eso es que a veces el paternalismo ha dañado la imagen, un poco perdiéndose la minga, pero sin embargo en algunas comunidades lo conservan. Así ha participado los cabildos, 
las comunas, las comunidades que era presidente, vicepresidente, tesorero o vocales de alcaldes que habia, los alcaldes eran quienes recorrían comunicando de casa en casa, para las reuniones o para mingas que tenían que realizarse.

Carolina: ¿decían el día, la hora, decían lo que se iba a hacer?

Remigio: ya con el profesor, participaban también los profesores en las mingas, al menos como vivía en la comunidad, pasaba el sábado el domingo en la comunidad, entonces trabajamos de forma conjunta organizando las comunidades y que se vea el trabajo, que no sea minga así por pasar un poco el tiempo, sino exigir el trabajo.

Carolina: ¿y las personas iban contentas para ver las mejoras en sus comunidades?

Remigio: bastante, a veces ellos mismos hacian su chicha para la minga, entonces hacian colaboración para la chicha, ahora no hay eso. En las mingas se ve solo colas pero no chicha, pero eso era más saludable.

Carolina: cuando se hacían las escuelas ¿habían niños de todas las edades?

Remigio: en la comunidad de Chiriguasi he tenido hasta alumnos de 14, 15 años porque yo fui a fundar esa escuela, se despertaron bastante por la educación, ya con la escuelita, en las comunidades, si de toda edad, de 6 años en adelante.

Carolina: ¿niñas y niños?

Remigio: niñas y niños, y más que todo había niños en la escuela de la Esperanza, estaban el primer grado, cuando yo me fui a Paniquimra les sacaron de la Esperanza y les pusieron en mi escuela. Ellos comparaban el trabajo que teníamos en las comunidades, con la escuela central de la parroquia; ellos evaluaban inclusive, decían acá está bueno, yo ya tenía 60 alumnos en el grado en primer grado, en la estrechera, y los padres inmediatamente para no verles así mandaron a hacer sillas, las mesitas para sus hijos para que estén cómodos hasta lograr conseguir las bancas. Igualmente Misión Andina dotaba de bancas, a las comunidades, material que era necesario, materiales didácticos que necesitamos en las comunidades. Misión Andina trabajaba sábados y domingos casi normalmente porque las mingas eran esos días, entre semana los padres de familia salían a sus trabajos fuera de la comunidad entonces no se podía exigir sino sábado y domingo.

Carolina: ¿cuál era el método de enseñanza y cuál era el curriculum?

Remigio: por ejemplo, en el normal Juan Montalvo nos decía que enseñáramos con palabras normales. En una comunidad ni siquiera saben el español, era difícil aplicar ese método, bien difícil. A nosotros inclusive para las prácticas, en las escuelas anexas al Juan Montalvo no nos permitían que practicáramos con cualquier otro método, sino método con palabras normales; entonces el profesor da en las comunidades, tenía que ingeniarse, a las comunidades el profesor que iba tenía que por lo menos saber algo de quichua. Yo apliqué el método silábico, entonces con el método silábico, y el método fonético se ha logrado que en un año el niño ya sabía leer y escribir, si ya sabía leer y escribir, igual recuerdo que nos sabía dar Misión Andina material didáctico con método silábico, con sus gráficos, entonces se ha podido lograr, mediante el método silábico. Los niños hablaban en quichua y yo traducía al castellano entonces se les facilitaba, mientras no se sabía el quichua era bastante dificil, los guaguas sabian puramente quichua, ahora como los niños ya hablaban en castellano, los padres ahora en las comunidades ya saben el quichua y el español.

Carolina: y por ejemplo, la relación del idioma y las personas que trabajaban en la Misión. Ellos conocían el idioma [quichua] o encontraban a alguien que pudiera ser traductor

Remigio: precisamente por falta de conocimiento del español eran explotados, por ejemplo los padres de familia o las madres de familia salían con sus ventitas a la ciudad, dígase para vender habas, trigo, cebada lo que sembraba en la comunidad, entonces no podían comunicarse y quien era consciente le pagaba bien, o alguien por ahi tenía que ayudar, por eso es que Misión Andina también habia seleccionado personal quichua que sepa para que vaya a las comunidades, caso contrario era bien dificil, ahora ya no hay este problema los indios saben, pero sin embargo los indios conversan en quichua entre ellos. 
Carolina: ¿usted tenía relación con la trabajadora social, hacían trabajos juntos?

Remigio: si, especialmente Misión Andina tenía trabajadora social que tenía que irse también a las comunidades y de hecho la trabajadora social también tenía que saber el idioma quichua para poder conversar en las comunidades para hacer las reuniones en las comunidades, entonces tenían trabajadora social para desarrollo comunitario, había médicos, había agrónomos, para hacer formar los huertos familiares en las comunidades.

Carolina: y la gente de las comunidades ¿cómo veía a los médicos?

Remigio: en ese entonces colaboraban participaban, recibian a la Misión Andina con gratitud y eran gratos también, eran buenos especialmente en las partes de Rumipamba, Siriguasa, Agra, Magdalena, Rinconada, haber que más, Chiriguasi San Clemente, son comunidades que pertenecen a la parroquia Esperanza del cantón Otavalo digamos del cantón Ibarra.

Carolina: Cuando llegaba la visita del médico, ¿las personas se hacían atender?

Remigio: sí, ellos acudían a esta atención médica, si acudian, a veces teníamos yo diría que establecer las dos medicinas la ancestral y la cientifica.

Carolina: ¿eso es compatible?

Remigio: dijéramos hasta el momento actual no se ha perdido de vista la medicina tradicional, la medicina ancestral. Si hay espacios donde que alguien llega por ahi, es afectado por fenómenos que le hacen daño a la salud. Le comento que había un doctor Luis Calderón especializado en Buenos Aires, era homeópata y él me comentaba que incluso él en calidad de médico tenía que hacerse una limpia y si alguna persona le traía algún paciente donde él, decía: "haga la limpia aqui, a lo mejor se hizo la limpia y luego se sanó y para qué va a venir donde mi", "yo no tengo ese poder" decía: "entonces si ya no se sanó entonces tráigale”. Si hay aquí una organización, precisamente de curanderos, de sachas que llamamos para curar, hay mucha gente que acude a la comunidad, que es organizado.

Carolina: claro, porque es otro sistema de salud.

Remigio: inclusive cuando se realizó la inauguración del subcentro de salud cuando fue Francisco Huerta Montalvo Ministro de Salud y tuvimos la oportunidad de hacer la demostración como un número, demostración de la curación con medicina ancestral y tradicional, pues él decía cómo sería bueno coordinar las dos la medicina ancestral y la científica. Por ejemplo en Otavalo, yo creo que están coordinando las dos medicinas, la ancestral y la científica.

Carolina:quizá recuerda otras personas que se hayan formado como líderes, como auxiliares de enfermería con la Misión

Remigio: si con la Misión Andina es que lo que aportaba ayudaba para que estudien y realmente si ha trabajado. Sino que a veces hubo ciertas cosas, comentarios que realmente, cuando yo no trabajaba con Misión Andina trabajaba con colegios, trabajaba con universidad. Aquí sí, hay que utilizar la palabra yo, aunque a veces no quiero, pero la verdad es que aquí soy casi el primer indígena que, y eso le iba a comentar que, en el colegio Juan Montalvo ya no me recibían por mi ropa, que se ponga terno, porque va a ser alumno maestro en quinto curso, en las prácticas que teníamos que hacer en las escuelas anexas, lo cual mi papá no sabía ni leer ni escribir, pero tenía mucha injerencia con las amistades, e injerencia en lo político. Mi papá se habia ido al Ministerio de Educación a avisar que no me reciben, entonces yo estaba esperando en la sala del rectorado del Juan Montalvo, llamó por teléfono y dijo que venga, ese rato fui, ahí entra y dice ya está matriculado y tiene que estar ahí, es un gusto que por primera vez en la historia un otavaleño haya venido al Juan Montalvo.

Carolina: ¿usted hasta que año trabajó con la Misión Andina?

Remigio: cuando estaba en Paniquimra, en Chiriguasi, 13 años he trabajado con Misión Andina.

Carolina: sería como hasta el 72.

Remigio: si porque en el 71 ya estaba por aquí formando el colegio de Quinchuquí

Carolina: le agradezco por su tiempo y por su apertura. 